\title{
Evaluation of Lead Hepatotoxicity; Histological, Histochemical and Ultrastructural Study
}

\author{
Ahmed M. S. Hegazy, Usama A. Fouad \\ Anatomy Department, Benha Faculty of Medicine, Benha University, Banha, Egypt \\ Email: ahmed0562301954@yahoo.com
}

Received 20 May 2014; revised 23 June 2014; accepted 4 July 2014

Copyright @ 2014 by authors and Scientific Research Publishing Inc.

This work is licensed under the Creative Commons Attribution International License (CC BY). http://creativecommons.org/licenses/by/4.0/

(c) (i)

\section{Abstract}

Lead is one of the most well-known naturally occurring environmental heavy metals. This experimental study was designed to evaluate lead induced toxic effects on hepatocytes and lobular architecture as judged microscopically. Material and Methods: This study was conducted in anatomy department, Benha faculty of medicine, Benha University, Egypt from May to October 2013 on 30 normal adult albino rats divided into 3 groups; one control and 2 experimental groups. The experimental groups were given $0.13 \%$ lead acetate solution in drinking water for 4 and 8 weeks, respectively. Animals were scarified and livers were removed and used to identify microscopic changes. Specimens were stained with Hematoxylin and eosin, with Masson trichrome stain for study of fibrous tissue and with periodic acid shiff's (PAS) to study the glycogen content .0ther specimens were prepared for ultrastructural study. Results: Mild lymphocytic infiltration, vacuolar degeneration and mild increase of periportal fibrosis with mild depletion of glycogen content and partial disappearance of glycogen vacuoles were reported in animals received contaminated water for 4 weeks. Animals maintained for 8 weeks on contaminated water showed hepatic changes in the form of abundant lymphocytic infiltration, increased cellular polymorphism, pyknotic nuclei and areas of cell necrosis with evident moderate periportal fibrosis and marked vacuolar degeneration associated with marked depletion of glycogen content. Ultrastructural study revealed mitochondrial edema, appearance of interstitial inflammatory cells, and appearance of scattered variable sized lead electron-dense inclusion bodies. Conclusion: It could be concluded that chronic exposure to lead imposes a potent toxic effect on liver cells manifested as glycogen depletion, cellular infiltration and liver architecture in the form of initiation of periportal fibrosis that may progress to liver cirrhosis.

\section{Keywords}

Lead, Rats, Liver, Light Microscope, Electron Microscope

How to cite this paper: Hegazy, A.M.S. and Fouad, U.A. (2014) Evaluation of Lead Hepatotoxicity; Histological, Histochemical and Ultrastructural Study. Forensic Medicine and Anatomy Research, 2, 70-79. 


\section{Introduction}

Nowadays, lead is usually absorbed into the body by drinking contaminated water or breathing polluted air. Water from lead pipes may be contaminated with lead. Fumes from lead-based paints, automobile exhaust, polluted air from industrial plants, or cigarette smoke may all contain lead. Some cosmetics even contain small amounts. Many lead-containing products, however, are now outlawed [1]. Childhood lead poisoning is an important, preventable environmental disease affecting millions of children around the world.

Children are at a higher risk than adults regarding effect of lead on health. This is partly due to higher gastrointestinal absorption and slower elimination resulting in a higher lead body burden in children. Children are also more prone to lead nephrotoxicity and neurotoxicity, especially lead encephalopathy which is the most severe form of lead poisoning [2].

Like other naturally occurring heavy metals_arsenic, mercury, and cadmium-lead also damages cellular substance and changes cellular genetics. The mechanism of lead toxicity, in general, involves oxidative damage that affects cell membrane and activates factors susceptible to transcription [3] [4].

The liver is divided into microscopic masses of cells functionally situated around terminal venules in an acinar unit. Blood flows from the terminal portal venules into the sinusoids and comes into contact with hepatocytes within the unit until it drains into the terminal hepatic venules. Hepatocytes, representing approximately 60\% of the liver cells, are 13 - $30 \mu$ in diameter and have eight or more surfaces. Each hepatocyte has both a microvillar and straight surfaces. The microvilli face the perisinusoidal space and extend into the pericellular space [5].

Hepatocyte cytoplasm typically displays much granular and a granular endoplasmic reticulum, many mitochondria, lysosomes and many well-developed Golgi bodes, features indicating a high metabolic activity. Glycogen granules and lipid vacuoles are usually prominent. Numerous, particularly large peroxisomes and vacuoles containing enzymes indicate complex metabolism in these cells [6].

Despite liver being not a target for lead toxic insult, [7] Wilczynska et al., (1998), conducted a retrospective observation covered 4.556 males with occupational disease, manifested by lead poisoning and reported increased total mortality among lead poisoned males with significant excess mortality was observed in diseases of the digestive system including chronic hepatitis and cirrhosis [7].

The mechanism of lead induced hepatotoxicity is not very clear [8].

This prospective experimental study was designed to evaluate the lead induced toxic effects on hepatocytes and lobular architecture as judged microscopically.

\section{Material \& Methods}

This study was conducted in anatomy department, Benha faculty of medicine, Benha University, Egypt from May to October 2013 on 30 normal healthy adult male albino rats purchased from the laboratories of Ministry of Agriculture. Rats were kept under standard conditions (temperature $20^{\circ} \mathrm{C}, 60 \%$ humidity and 12 hours day/night cycle), and maintained on standard diet and pure distilled water till start of study regimens.

The animals were divided into three equal groups each of 10 rats. Control group rats were kept on normal diet and pure distilled water (Group A). The 2 experimental groups were kept on normal diet and given a $0.13 \%$ lead acetate solution in drinking water [9] for 4 weeks (Group B) and 8 weeks (Group C) respectively. Rats were grown each group in a separate cage under standard conditions throughout the study period.

Then, animals were sacrificed by decapitation and livers were removed. For histological \& histopathological examinations small pieces of the liver were fixed in $10 \%$ neutral formalin \& prepared for Hematoxylin \& Eosin stain, and trichrome stain [10] which stains the fibrous tissue. For histochemical study, other specimens were fixed in alcoholic bouin's fluid for glycogen demonstration using [11] periodic acid shiff's (PAS) technique.

For electron microscopic study, small slices of liver were fixed immediately in $2.5 \%$ gluteraldehyde with 0.1 M phosphate buffer, dehydrated and observed with light microscope. Selected areas were noted and ultra thin sections of (70 - $100 \mathrm{~nm}$ ) were cut using the ultramicrotome then stained with 2 - 1 uranyl acetate and lead citrate for 30 minutes each of them observed \& photographed with Jeol 100 electron microscope.

\section{Results}

\subsection{Histopathological Observations}

Hepatic specimens obtained from the control group showed normal architecture of hepatic tissue with normal 
hepatocytes each cell is polygonal in shape with a large rounded vesicular nucleus inside an acidophilic cytoplasm. These hepatocytes are arranged in cords radiating from the central vein and are separated by irregular sinusoids (Figure 1).

Specimens taken from rats given $0.13 \%$ lead acetate solution in drinking water for 4 weeks (short duration) showed mild infiltration with inflammatory cells adjacent to the vein which appeared congested. The hepatocytes showed degeneration of cytoplasm which appeared vacuolated (Figure 2). Specimens examined from liver sections taken from rats exposed for 8 weeks (long duration) showed more affection of the hepatic lobules manifested by loss of hepatic architecture, pyknotic nuclei, some sections showed more affection in the form of hepatic cell necrosis (Figure 3 ).

Control liver specimens stained by Masson trichrome stain showed normal thin layer of periportal fibrous tissue with appeared bluish in color (Figure 4). Specimens treated with lead acetate for short duration (4 weeks) showed mild increase in the amount of periportal fibrous tissue (periportal fibrosis) which is also seen radiating in between the cords of hepatic cells (Figure 5). Moreover, specimens examined after 8 weeks of lead acetate administration (long duration) showed moderate increase in the amount of the periportal fibrous tissue and also radiating in between the cords of hepatocytes (Figure 6).

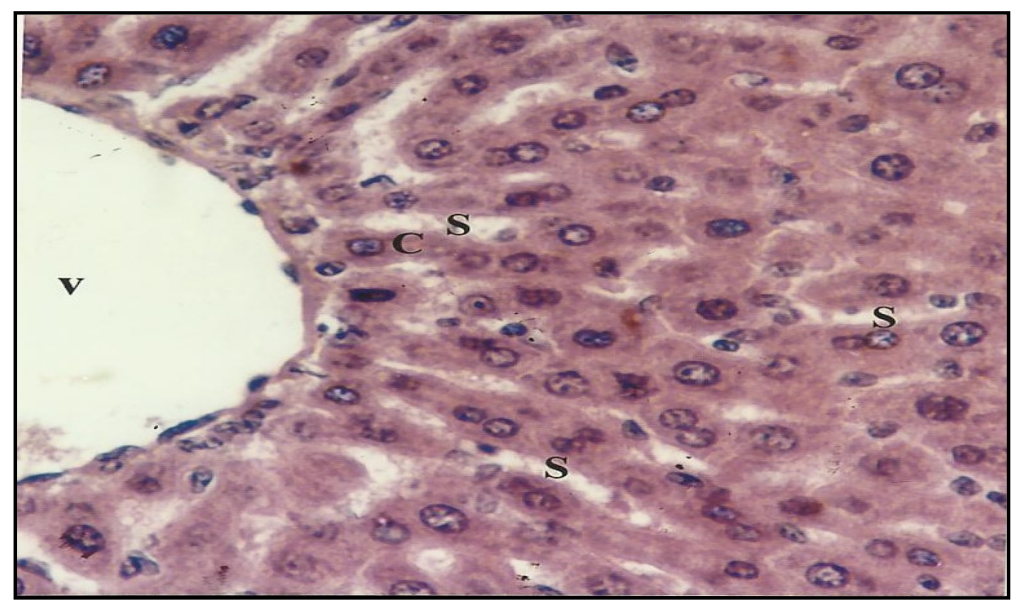

Figure 1. A photo micrograph of a liver section of a rat in the control group showing: normal central vein (V) normal arrangement in of liver cords. The hepatocytes (C) are seen radiating from the central vein and separated from each other by normal irregular blood sinusoids (S) (Hx \& E. × 400).

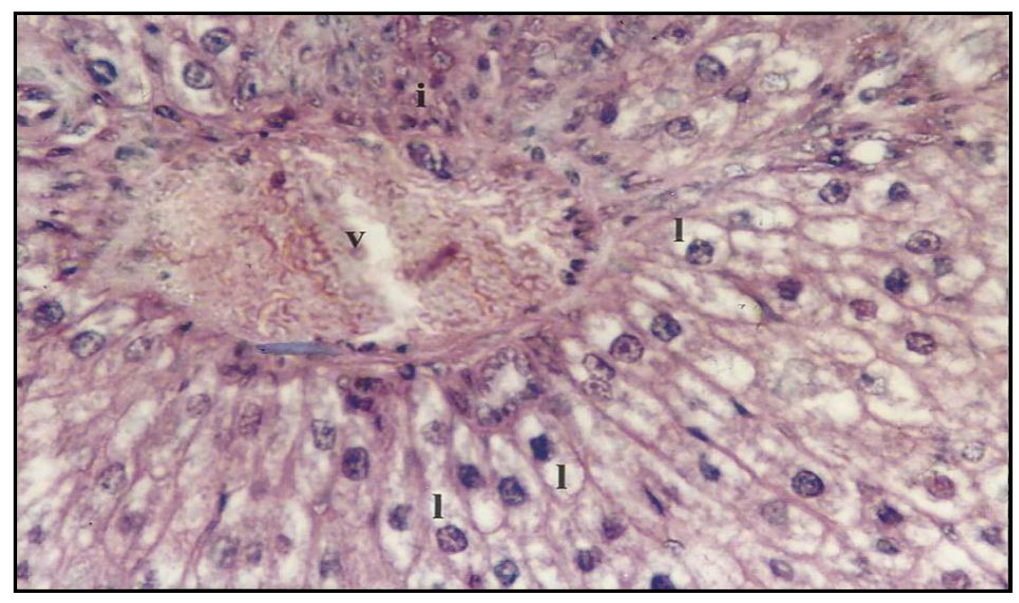

Figure 2. A photomicrograph of a liver section of a rat given $0.13 \%$ lead acetate solution in drinking water for 4 weeks (short duration) showing: mild infiltration with inflammatory cells (i) near the central vein (V) which is congested. Vacuolated hepatocytes (l) are seen (Hx \& E. × 400). 


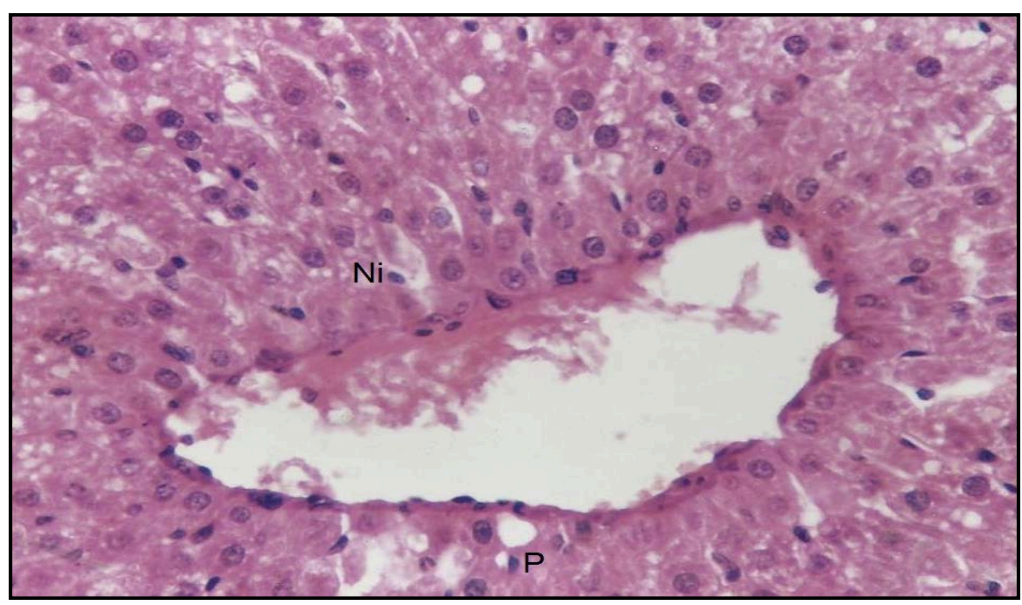

Figure 3. A photomicrograph of a liver section of a rat given $0.13 \%$ lead acetate solution in drinking water for 8 weeks (long duration) showing loss of liver architecture, with areas of necrosis (Ni). Pyknotic nuclei $(\mathrm{P})$ seen $(\mathrm{Hx} \& \mathrm{E} . \times$ 400).

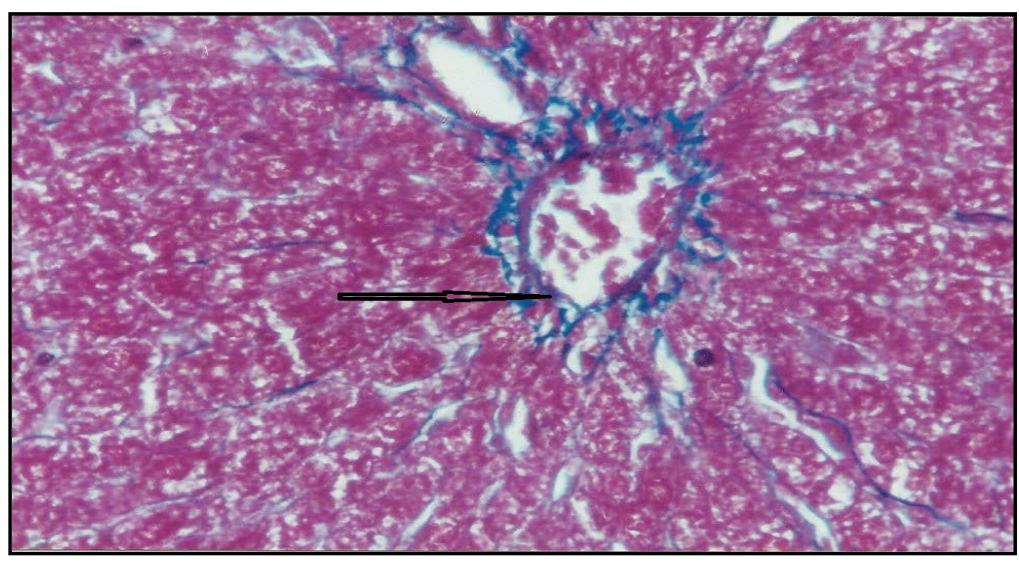

Figure 4. A photo micrograph of a liver section of a rat in the control group showing: normal thin layer of periportal bluish fibrous tissue (arrow) (Masson Trichrome $\times 400)$.

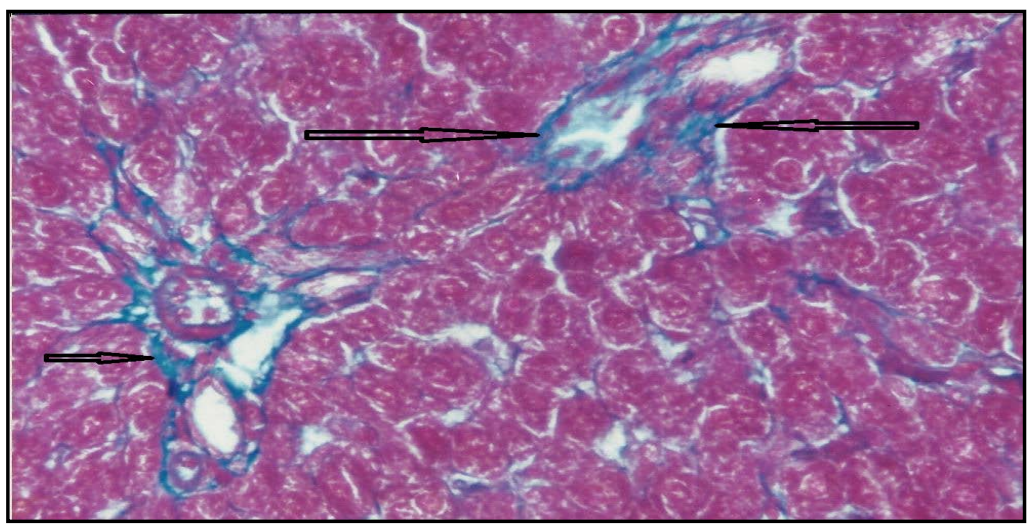

Figure 5. A photomicrograph of a liver section of a rat given $0.13 \%$ lead acetate solution in drinking water for 4 weeks (short duration) showing: mild increase of the periportal fibrosis (arrows) which is also seen radiating in between the cords of hepatocytes (Masson Trichrome $\times 400$ ). 


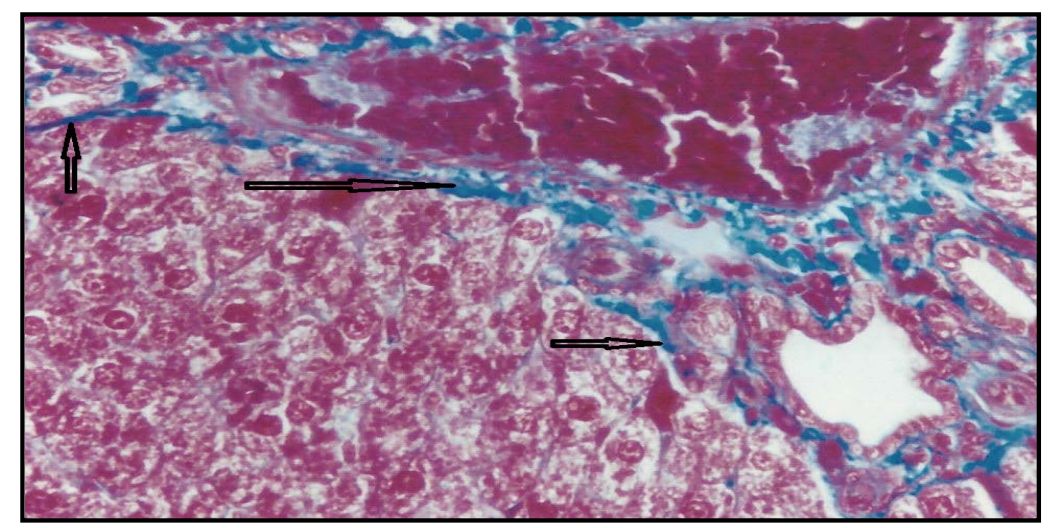

Figure 6. A photomicrograph of a liver section of a rat given $0.13 \%$ lead acetate solution in drinking water for 8 weeks (long duration) showing: moderate increase of the periportal fibrosis (arrows) radiating also in between the cords of hepatic cells (Mason Trichrome $\times 400$ ).

\subsection{Histochemical Observations}

Control hepatic specimens stained with PAS showed normal content of glycogen particles which appeared as deeply red purple colored PAS-positive inclusions densely located inside the cytoplasm (Figure 7).

Specimens treated with lead acetate for short period showed mild glycogen depletion involving many hepatic cells (Figure 8). Those specimens treated for a longer duration (8 weeks) showed moderate glycogen depletion especially in the central area of hepatic cells radiating from the central vein (Figure 9)

\subsection{Ultrrastructural Observations}

EM examination of control specimens revealed normal hepatocytes. Each hepatocyte is characterized by a prominent round nucleus normal mitochondrion, ribosomal studded-endoplasmic reticulum and normal bile canaliculus separating one hepatocyte from the adjacent one (Figure 10).

Specimens taken from rats treated with lead acetate for a short period showed heavy infiltration with electron dense (lead) particles which appeared as dark spots interstitial inflammatory cells are also seen (Figure 11). The mitochondria became swollen in addition to scattered cytoplasmic lysosomal inclusion bodies (Figure 12 \& Figure 13).

\section{Discussion}

African children are particularly predisposed to environmental lead exposure, because of their life style and socioecological factors. Gasoline sold in most African countries contains 0.5 - $0.8 \mathrm{~g} / 1$ lead. In urban and rural areas and near mining centers, average lead concentrations reach $0.5-3.0 \mathrm{mu} \mathrm{g} / \mathrm{m}^{3}$ in the atmosphere and 1000 $\mathrm{mug} / \mathrm{g}$ in dust and soils. In addition to automotive and industrial sources, cottage industries and the burning of paper products, discarded rubber, battery casings and painted woods for cooking and heading represent additional hazards to individual households. Lead paint, lead solder and lead cosmetics are unregulated in some countries [12].

Lead is a poison that affects virtually every system in the body.

Children are more vulnerable to lead exposure than adults because of the frequency of pica, hand- to -mouth activity, and a greater rate of intestinal absorption and retention, Lead has a potent preferential susceptibility of the highly vascularized organs and even organized compartments [13].

The mechanism of Lead induced hepatictoxicity is not very clear. Results from some recent studies intensely propose that oxidative stress and cellular apoptosis are the main causes for hepatic pathophysiology [14] [15].

In the present study, liver specimens taken 4 weeks after exposure to lead showed minimal inflammatory cellular infiltration and starting vacuolation of hepatocytes, despite this picture is not characteristic for lead poisoning it reflected the body burden imposed by administration of a poisonous substance. This goes in hand with Gunawan \& Kaplowitz, (2004), [16] who stated that most adverse hepatic reactions to xenobiotics require me- 


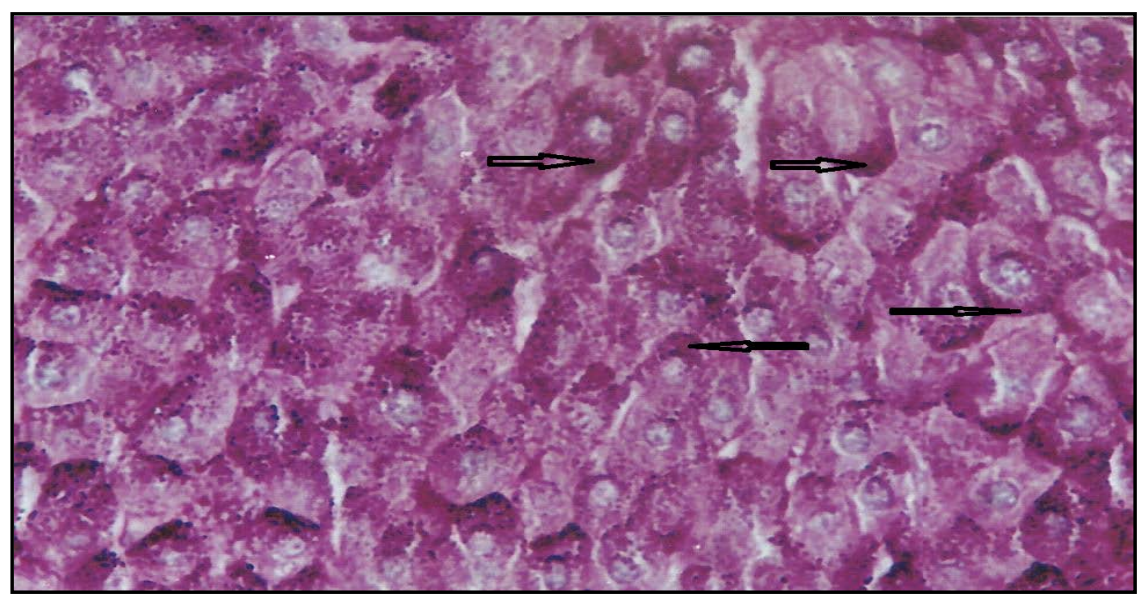

Figure 7. A photo micrograph of a liver section of a rat in the control group showing: normal content of glycogen as deeply purple colored PAS-positive inclusions densely located in the cytoplasm (arrows) (PAS $\times 400)$.

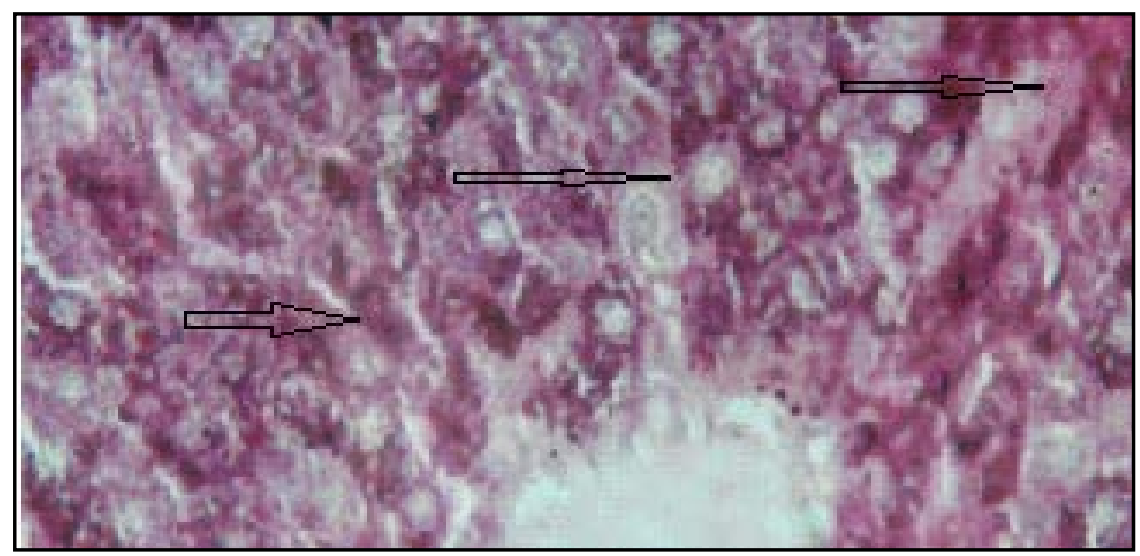

Figure 8. A photomicrograph of a liver section of a rat given $0.13 \%$ lead acetate solution in drinking water for 4 weeks (short duration) showing: Mild glycogen depletion in the majority of the hepatocytes (arrows) (PAS $\times 400)$.

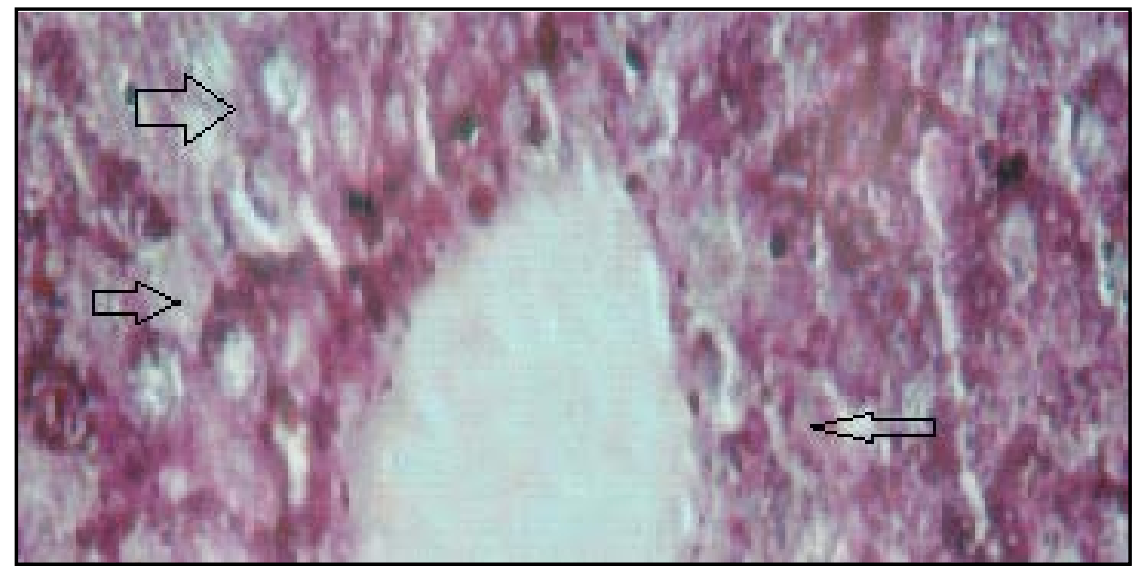

Figure 9. A photomicrograph of a liver section of a rat given $0.13 \%$ lead acetate solution in drinking water for 8 weeks (long duration) showing: moderate glycogen depletion involving especially the central area of hepatic cells radiating from the central vein (V) (arrows) (PAS $\times 400)$. 


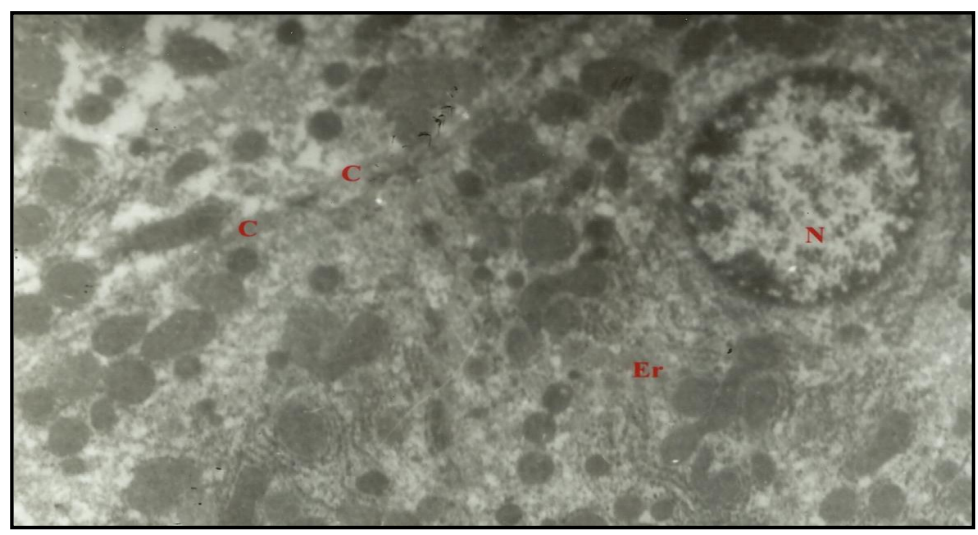

Figure 10. An electron photo micrograph of a liver cell from a control rat showing: normal mitochondria $(\mathrm{C})$, prominent rounded nucleus $(\mathrm{N})$, ribosomal studded-endoplasmic reticulum $(\mathrm{Er})$. Original magnification $\times 4000$.

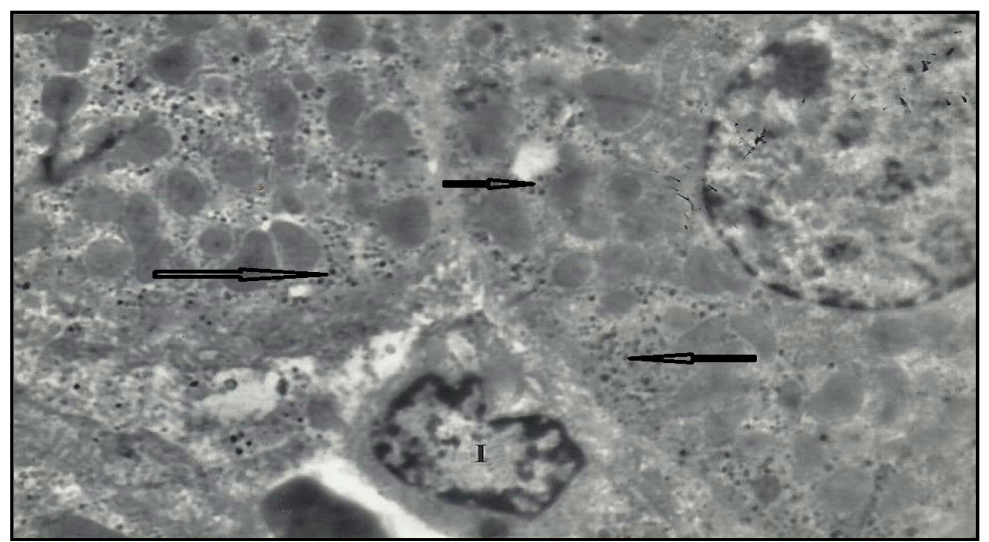

Figure 11. An electron photomicrograph of a liver cell of a rat given $0.13 \%$ lead acetate solution in drinking water for 4 weeks. (Short duration) showing: heavy infiltration with electron-dense (lead) particles which appeared as dark spots (arrows) together with an interstitial inflammatory cell (I). Original magnification $\times 6000$.

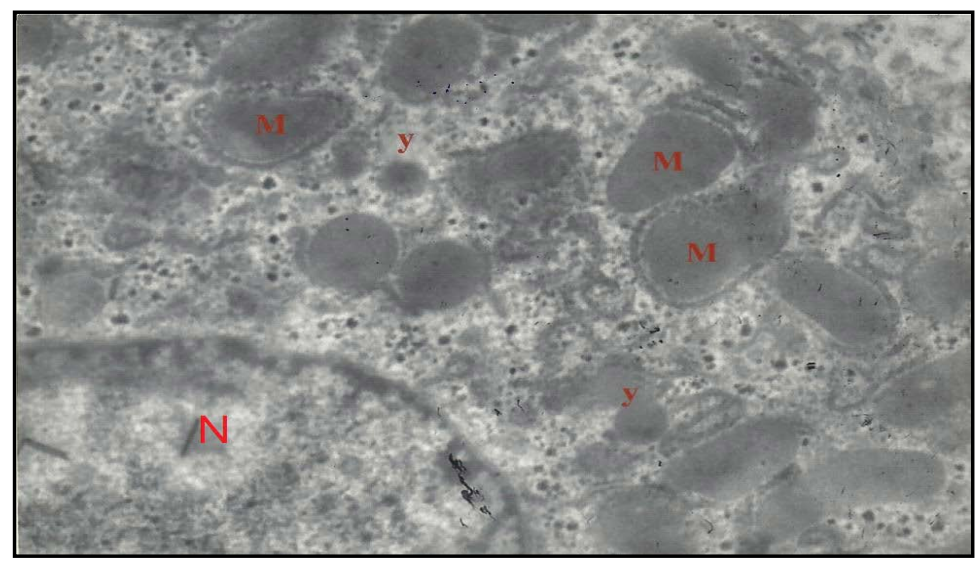

Figure 12. An electron photomicrograph of a liver cell of a rat given $0.13 \%$ lead acetate solution in drinking water for 4 weeks. (Short duration) showing: the mitochondria are clearly swollen $(\mathrm{M})$ in addition to the presence of lysosomal inclusion bodies (y) scattered in the cytoplasm especially near the nucleus $(\mathrm{N})$. Original magnification $\times 10,000$. 


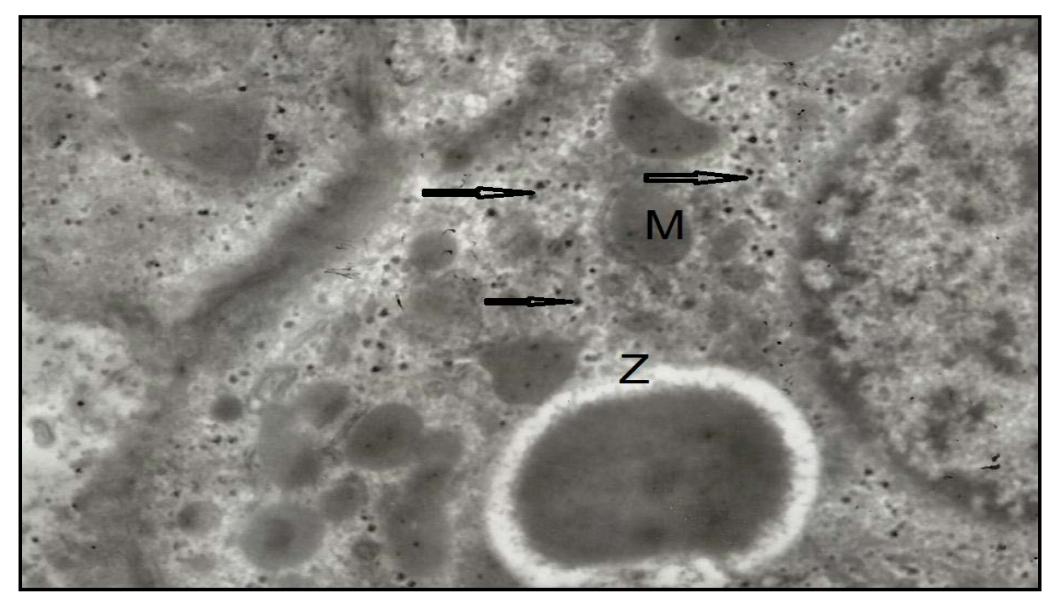

Figure 13. An electron photomicrograph of a liver cell of a rat given $0.13 \%$ lead acetate solution in drinking water for 8 weeks. (Long duration) showing: the presence of a large dense particle surrounded by a clear zone $(Z)$ involving a large area of the cytoplasm adjacent to the nucleus in addition to mitochondrial swelling $(\mathrm{M})$ and heavy infiltration with electron-dense dark spots (arrows) (lead particles). Original magnification $\times 10,000$.

tabolism of the drug to reactive metabolites and free radicals, which then either lead to direct overwhelming lethal insult, nonlethal sensitization to the lethal effects of the innate immune system, or haptenization eliciting an immunoallergic response of the adaptive. Immune system manifested by inflammatory cellular infiltration and activation of apoptotic systems. The results in this study go in hand with (Pal et. al., 2013) [17] who stated that lead has been reported to cause liver injury and cellular apoptosis.

Some hepatocytes showed cytoplasmic vacuoles, nuclear pyknosis and complete cellular necrosis in the rats treated for along period with lead. Such a change was previously demonstrated by Bennett, (1985) and Corpas et al., (1996) [18] [19]. Also, these histological picture coincided with that detected in avian study conducted by Kertesz \& Fancsi (2003) [20] who reported that liver of ducklings exposed to surface water pollution with lead and other heavy metals showed significant lesions, dystrophy in the form of hepatic necrosis was detected in each treated animal.

In our study, liver specimen showed irregular hepatocytes with evident periportal fibrosis and marked vacuolar degeneration with depletion of glycogen content especially in those treated with lead for a longer duration. Russo et al., (1988) [21] studied the effects of lead on the kidneys and liver of rats given lead acetate in their drinking water for 6 months and reported that liver damage by lead was largely confined to centrilobular regions. Endothelial and Kupffer cells were the most affected; they often sequestered large numbers of the particles. In parenchymal cells, particles were few and mainly in vesicles, but they were more widely distributed in the cytoplasm. Ellender \& Ham (1987) [22] studied the effects of lead on connective tissue and reported that lead induced local lesions characterized by changes in granulation tissue and approximating connective tissue. The granulation tissue contained mononuclear phagocytes in various guises, and some cells with intranuclear inclusion bodies and the matrix of the granulation tissue contained collagen fibrils having a wide range of diameters suggestive of altered collagen biosynthesis. That foci of collagen mineralization occurred in zones of combined trauma and lead impregnation. These foci once mineralized became enveloped by giant cells and epitheloid cells. These studies could explain the reported periportal fibrosis and hepatocyte disfigurement recorded in our study.

Moreover, specimens stained with PAS showed mild depletion of glycogen content after 4 weeks and moderate depletion after 8 weeks.

The toxic effects of lead on the liver detected by the light microscope in the present study agreed with Villa et al., (1993), [23] who studied the toxic effect of lead on liver and spleen of rats using light microscopy and reported that lesions and lead deposits confirmed that lead is the causative agent.

Similarly, Corpas et al., (1996) [19] studied the effects of lead on hepatic and renal tissue of rats exposed to lead and reported lead intranuclear bodies and cytoplasmic vacuoles.

Papaioannou et al., (1998) [24] reported that intranuclear inclusion bodies is the most characteristic and pa- 
thognomonic finding induced by lead intoxication. They described lead inclusion bodies as bodies with a dense central core enveloped in a cortex of matted and radiating filaments and found them in all their sacrificed rats. In our study we observed such inclusion bodies in the juxta nuclear cytoplasm just adjacent and outside the nuclear membrane as evident by ultrastructural examination of the specimens examined after a long period of administration. Villa et al., (1993) [23] attributed the presence of these inclusion bodies in liver and spleen specimens of rats exposed to lead toxic doses by the fact that these organs are considered to harbor a great amount of constant macrophages with phagocytic function.

Ceruti et al., (2002) [25] reported characteristic histological alterations ascribed to lead in wild rats exposed to high levels of lead and reported that the incidence of intranuclear inclusion bodies and karyocytomegally was higher in rats with high lead levels and in those exposed to lead for longer durations.

Goering \& Fowler, (1985) [26] thought that inclusion bodies represent binding of lead by a binding protein that may mitigate the effects of lead on the cell's function.

Jarrar \& Mahmoud, (2000) [27] studied lead intranuclear bodies that have resulted in the kidneys of adult Wister male rats receiving lead acetate in their diet using histological and histochemical techniques to determine their nature and reported that intranuclear inclusion bodies proved to be lead lipoprotein complexes containing sulfhydry1 groups and are basic in nature.

In the present study, the mitochondria became swollen in the long term treated group. These results go in hand with (Pal et. al., 2013) [17] who proved that Lead leads to hepatic cellular apoptosis via mitochondria dependent pathway.

\section{Conclusion}

It could be concluded that chronic exposure to lead imposes a potent toxic effect on the liver cells and architecture manifested as glycogen depletion, cellular infiltration, cellular necrosis and initiation of periportal fibrosis that may progress to liver cirrhosis.

\section{Recommendations}

It is recommend that, the industrial companies which extract or use lead in different industrial processes must tightly protect the workers in such places. Moreover, the environmental lead pollution and uncontrolled industrial use of lead must be controlled.

\section{References}

[1] Moreira, F.R. and Moreira, J.C. (2004) Effects of Lead Exposure on the Human Body and Health Implications. Revista Panamericana de Salud Pública, 15, 119-129. http://dx.doi.org/10.1590/S1020-49892004000200007

[2] Meyer, P.A., McGeehin, M.A. and Falk, H. (2003) A Global Approach to Childhood Lead Poisoning Prevention. International Journal of Hygiene and Environmental Health, 206, 363-369.

[3] White, L.D., Cory-Slechta, D.A., Gilbert, M.E., TiVany-Castiglioni, E. and Zawia, N.H. (2007) New and Evolving Concepts in the Neurotoxicology of Lead. Toxicology and Applied Pharmacology, 225, 1-27. http://dx.doi.org/10.1016/j.taap.2007.08.001

[4] Ahamed, M. and Siddiqui, M.K.J. (2007) Low Level Lead Exposure and Oxidative Stress: Current Opinions. Clinica Chimica Acta, 383, 57-64. http://dx.doi.org/10.1016/j.cca.2007.04.024

[5] Jones, A.L. (1982) Anatomy of the Normal Liver. In: Hepatology, Z.D. and Boyer, T.D., Eds., Hepatology. A Text Book of Liver Disease, W.B. Saunders Co, Philadelphia, 3-31.

[6] Bannister, L.H. (1995) Alimentary System. In: Banister, L.H., Berry, M.M., Collis, P.P., Dyson, M., Dussek, J.E. and Ferguson, M.W., Eds., Grays’s Anatomy, 38th Edition, Churchill Livingstone, London, 1683-812.

[7] Wilczynska, U., Szeszenia-Dabrowska, N. and Sobala, W. (1998) Mortality of Men with Occupational Lead Poisoning in Poland. Medycyna Pracy, 49, 113-128.

[8] Sharma, V., Sharma, S., Pracheta and Sharma S (2011) Lead Induced Hepatotoxicity in Male Albino Mice: The Protective Potential of the Hydromeyhanolic Extract of Withania Somnifera. International Journal of Pharmaceutical Sciences Review and Research, 7, 116-121.

[9] Mahmoudzadeh Sagheb, H.R., Heidari, Z., Barbarestani, M. and Noori Mugahi, M.H. (2004) Ultrastructural Study of Neutrophils in Fetal Rat Spleen Following Lead Intoxication. Yakhteh Medical Journal, 5, 172-177.

[10] McManus, J. and Mowry, R. (1963) Staining Methods. Histological and Histochemical Harber and Row Inc., New 
York and London.

[11] Hotchkiss, R.D. (1948) A Microchemical Reaction Resulting in the Staining of Polysaccharide Structures in Fixed Tissue Preparations. Archives of Biochemistry, 16, 131.

[12] Nriagu, J.O., Blankson, M. and Ocran, K. (1996) Childhood Lead Poisoning in Africa: A Growing Public Health Problem. Science of the Total Environment, 181, 93-100. http://dx.doi.org/10.1016/0048-9697(95)04954-1

[13] Srianujata, S. (1998) Lead-The Toxic Metal to Stay with Human. The Journal of Toxicological Sciences, Suppl. 2, 237240. http://dx.doi.org/10.2131/jts.23.SupplementII_237

[14] Sharma, V. and Pandey, D. (2011) Protective Role of Tinospora ordifolia against Lead-Induced Hepatotoxicity. Toxicology International, 17, 12-17. http://dx.doi.org/10.4103/0971-6580.68343

[15] Clement, G.Y. and Paul, B.T. (2007) N-Acetyl-L-Cysteine Affords Protection against Lead-Induced Cytotoxicity and Oxidative Stress in Human Liver Carcinoma (HepG2) Cells. International Journal of Environmental Research and Public Health, 4, 132-137. http://dx.doi.org/10.3390/ijerph2007040007

[16] Gunawan, B. and Kaplowitz, N. (2004) Clinical Perspectives on Xenobiotic-Induced Hepatotoxicity. Drug Metabolism Reviews, 36, 301-312. http://dx.doi.org/10.1081/DMR-120034148

[17] Pal, P.B., Sinha, K. and Sil, P.C. (2013) Mangiferin, a Natural Xanthone, Protects Murine Liver in Pb (II) Induced Hepatic Damage and Cell Death via MAP Kinase, NFkB and Mitochondria Dependent Pathways. PLoS One, 8, Article ID: e56894. http://dx.doi.org/10.1371/journal.pone.0056894

[18] Bennett, W.M. (1985) lead Nephropathy. Kidney International, 28, 12-20. http://dx.doi.org/10.1038/ki.1985.143

[19] Corpas, I., Benito, M.J. and Antonio, M.T. (1996) Hepatic and Renal Alterations in Newborn Rats Induced by Prenatal and Early Lactational Exposure to Lead and/or Cadmium. Reviews in Toxicology, 13, 76-82.

[20] Kertesz, V. and Fancsi, T. (2003) Adverse Effects of (Surface Water Pollutants) Cd, Cr and Pb on the Embryogenesis of the Msallard. Aquatic Toxicology, 65, 425-433. http://dx.doi.org/10.1016/S0166-445X(03)00155-3

[21] Russo, M.A., Kapoor, S.C. and van Rossum, G.D. (1988) Localization of Lead in the Kidney and Liver of Rats Treated in Vivo with Lead Acetate: Ultrastructural Studies on Unstained Sections. British Journal of Experimental Pathology, 69, 221-234.

[22] Ellender, G. and Ham, K.N. (1987) Connective Tissue Responses to Some Heavy Metals. I. Sodium Loaded Ion Exchange Beads as a Control: Histology and Ultrastructure. British Journal of Experimental Pathology, 68, 277-289.

[23] Villa, N.R., Mercau, G., Riera, N.M., Santos, N.S. and Vitalone, H. (1993) Lead: Histopathological Findings in Experimental Contamination. Acta Gastroenterol Latinoam, 23, 159-163.

[24] Papaioannuo, N., Vlemmas, I., Balaskas, N. and Tsangaris, Th. (1998) Histopathological Lesion in Lead Intoxicated Dogs. Veterinary and Human Toxicology, 40, 203-207.

[25] Ceruti, R., Ghisleni, G., Ferretti, E., Cammarata, S., Sonzogni, O. and Scanziani, E. (2002) Wild Rats as Monitors of Environmental Lead Contamination in the Urban Area of Milan, Italy. Environmental Pollution, 117, 255-259. http://dx.doi.org/10.1016/S0269-7491(01)00273-1

[26] Goering, P.L. and Fowler, B.A. (1985) Mechanism of Renal Lead-Binding Protein Reversal of Delta-Aminolevulinic Acid Dehydratase Inhibition by Lead. Journal of Pharmacology and Experimental Therapeutics, 234, 365-371.

[27] Jarrar, B.M. and Mahmoud, Z.N. (2000) Histochemical Characterization of the Lead Intranuclear Inclusion Bodies. Biological Trace Element Research, 75, 245-251. http://dx.doi.org/10.1385/BTER:75:1-3:245 